\title{
Sicher auch bei interventionellen Eingriffen
}

_ Das orale Antikoagulans Rivaroxaban $\left(\right.$ Xarelto $\left.^{\circ}\right)$ ist eine wirksame Therapieoption in mehreren Indikationen, wobei die Schlaganfallprophylaxe bei Vorhofflimmern (VF) sowie die Behandlung und Sekundärprophylaxe venöser Thromboembolien die wichtigsten darstellen. Aber auch im Rahmen folgender interventioneller kardiologischer Verfahren ist Rivaroxaban gut untersucht und sicher.

1 KHK-Patienten mit VF, die einen Stent bekommen (PIONEER AFPCI): Die 2.124-Patienten-Studie verglich in dieser Situation zwei Antikoagulationsstrategien auf Rivaroxaban-Basis mit dualer Plättchenhemmung plus Warfarin. In beiden Rivaroxaban-Armen war das Blutungsrisiko signifikant niedriger $(16,8 \%$ bzw. $18 \%$, vs. $26,7 \%$, relatives Risiko (RR) $41 \%$, p < 0,001). Bei der Effekti- vität ergaben sich keine Unterschiede. Die Daten legen nahe, dass ein Rivaroxaban-Regime, z. B. 15 mg/d plus ein P2Y $\mathrm{Y}_{12}$-Inhibitor wie Clopidogrel für 12 Monate, die bevorzugte Therapie sein sollte, sagte Prof. Christoph Bode, Freiburg, der an der Studie beteiligt war.

2 KHK-Patienten ohne VF mit akutem Koronarsyndrom (ACS): Die Studie ATLAS ACS-2 TIMI 51 untersuchte den Wert einer niedrigdosierten Rivaroxaban-Nachbehandlung bei 15.526 ACS-Patienten vs. Placebo. $2 \times 2,5 \mathrm{mg} / \mathrm{d}$ reduzierten das relative Risiko für Herztod, Herzinfarkt und Schlaganfall um $16 \%(8,9 \%$ vs. $10,7 \%)$ und die Gesamtmortalität $(2,7 \%$ vs. $4,1 \%)$.

3 Patienten mit VF, bei denen eine Ablation durchgeführt wird (VENTURE-AF): Wenn bei VF unter Antikoagulation abladiert wird, geschieht das mit Rivaroxaban (20 $\mathrm{mg} / \mathrm{d}$ ) ebenso sicher wie mit einem Vitamin-K-Antagonisten (VKA) - so das Ergebnis dieser Studie mit 248 Patienten.

4 Patienten mit VF, bei denen eine Kardioversion durchgeführt wird (X-VeRT]: Im Rahmen einer elektiven Kardioversion von VF ist Rivaroxaban eine effektive und sichere Alternative zu VKA, so das Ergebnis dieser Studie mit 1.504 Patienten. Thromboembolische Komplikationen waren insgesamt selten $(0,51 \%$ Rivaroxaban, $1,02 \%$ VKA), ebenso schwere Blutungen (0,6\% Rivaroxaban, $0,8 \%$ VKA).

\section{Dr. Dirk Einecke}

- Presseveranstaltung "Info-ExPRESS: Fakten, Fälle, und Facetten zur Antikoaguation mit Xarelto ${ }^{\oplus}$ bei interventionellen kardiologischen Verfahren"; Frankfurt, Februar 2017 (Veranstalter: Bayer Vital)

\section{Moderne Arthrosetherapie}

\section{Ernährung und Sport im Fokus}

_ Arthrose ist weltweit die häufigste Gelenkerkrankung bei Erwachsenen. Und die betroffenen Patienten werden immer jünger, berichtete die Medizinerin Margret Ulrich vom Unternehmen Orthomol.

Bei einer Arthrose wird durch die degenerativen Prozesse im Gelenk die Versorgung des Knorpels beeinträchtigt, so Ulrich. Hier kann eine ausgewogene Ernährung, die unter anderem durch Knorpelbestandteile wie Glucosaminund Chondroitinsulfat, Hyaluronsäure und Kollagenhydrolysat ergänzt wird, sinnvoll sein.

Bestätigt wurde der Nutzen einer ergänzenden bilanzierten Diät mit einer Nährstoffkombination (Orthomol arthroplus ${ }^{\circledast}$ ) in einer placebokontrollierten Doppelblindstudie mit insgesamt 366
Patienten mit symptomatischer Kniegelenksarthrose. Die Patienten erhielten über einen Zeitraum von drei Monaten täglich eine Tagesportion der Nährstoffkombination oder Placebo.

Das Ergebnis: Der Responder-Anteil lag in der Verumgruppe nach drei Monaten bei $59,4 \%$ vs. $43,8 \%$ in der Placebogruppe ( $\mathrm{p}=0,008)$. Als Responder galten Patienten, bei denen eine Verbesserung des Lequesne-Index um mindestens 3 Punkte und zugleich das ärztliche Globalurteil "gebessert" oder „sehr gebessert“ erreicht wurde. Um den Knorpel ausreichend mit Nährstoffen zu versorgen, müssen die Mikronährstoffe aus der Gelenkflüssigkeit in den Knorpel eingewalkt werden. Hierzu ist Bewegung unerlässlich. Hier sollte bei Arthrosepatienten der Schwerpunkt auf

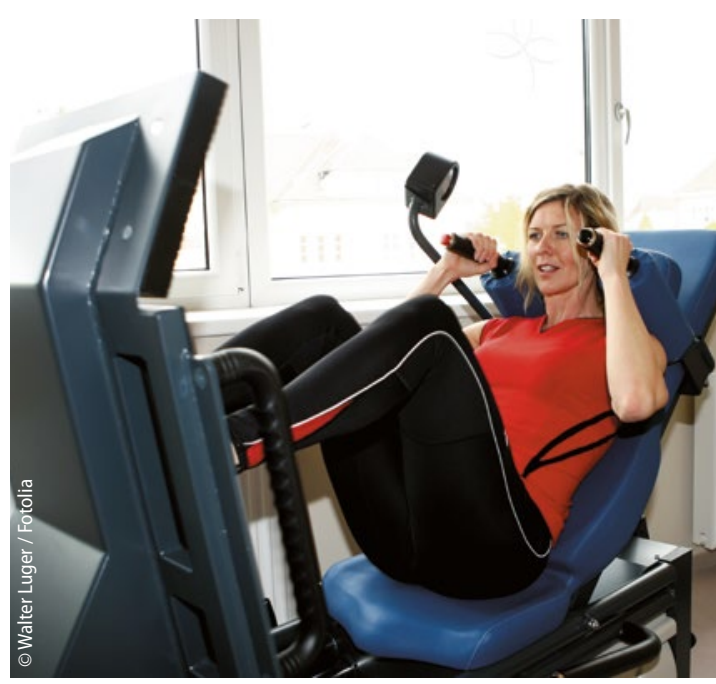

Starke Muskeln entlasten die Gelenke.

einem vernünftig durchgeführten Krafttraining liegen.

Christina Ott

- Update Arthrosemanagement "Effektive Therapiebegleitung mit Ernährungs- und Sportmedizin"; Köln, Mai 2017 (Veranstalter: Orthomol) 